\title{
Manipulation of Attention in Children with Reading
}

\author{
Roann Munoz Ramos
}

\begin{abstract}
The skill of reading is crucial in order to advance in society. However, for about a tenth of children, learning to read can demand from them more considerable efforts. Having considerable reading problems or dyslexia interferes with learning and academic success. Aside from phonological difficulties, reading problems have been linked to attention deficits, such as auditory and visual spatial attention. This research attempts to assess attention of a select group of children with and without reading problems as indicated by ear advantage results in the dichotic listening tasks. Results show that normal readers have a relatively stable baseline REA and in the ViDiLi Non-forced dichotic listening task; however, their ear advantage can be affected by explicit instructions to focus on a particular ear during dichotic listening, as indicated by a visually-guided dichotic listening task. On other hand, children with reading problems exhibit unstable ear advantage in all three dichotic listening activities. The greater variability in the ear advantages reported by the reading problem group in the bimodal tasks may indicate the influence of visual cues in their ability to switch attention.
\end{abstract}

Index Terms-Attention, ear advantage, children with reading problems.

\section{INTRODUCTION}

The concept of attention has been a topic of interest among philosophers and psychologists. However, it was during the early years of experimental psychology that shaped the views and approaches regarding attention. Current understanding of attention has broadened with the emergence of imaging techniques and computer technology.

Because of the generic nature of attention, which covers not only a single concept, there is no coherent field of attention from either a psychological or a physiological viewpoint. It is for this reason that attention is considered to be an "umbrella" term that refers to many separate cognitive processes. An example is selective attention, which exists in every sensory modality. While the early selective attention studies focused on studying sensations separately, multi-sensory researches, which combine tasks of different sensory media (e.g., auditory and visual), have examined whether directing attention for a task in a particular sense organ may have consequences for processing in other modalities. A key finding in the cross modal spatial attention effect is that the direction of spatial attention for a task in one modality can modulate sensory-specific processing for another modality to produce cross-modal influences at stages that would be considered "uni-modal." [1]. According to the modality appropriateness explanation, different sensory modalities are specialized to process the senses' respective information. The most applicable sensory channel is selected

Manuscript received February 25, 2013; revised May 20, 2013.

R. M. Ramos is with the University of Santo Tomas, Espana Blvd, Manila, Philippines (e-mail: roann.ramos@gmail.com). depending on the task's nature. Sensory conflict experiments conclude there is no complete domination of the senses; rather, information from the senses is integrated while evidence from the modality most suitable for the current activity is given more weight. Focus of attention depends on which sense organ is likely to provide the most accurate and suitable information for the required task.

A common form of childhood disorder, dyslexia is a learning disorder primarily characterized by reading problems despite possessing adequate intelligence and schooling. Domain-specific definitions of learning disorders have been developed. One classification as to the causes of dyslexia points out to its four major deficits: (a) language processing deficits; (b) memory deficits; (c) perceptual deficits; and, (d) attention impairments.

As a specific language-based disorder, dyslexia is characterized by difficulties in single word decoding which is reflective of an insufficient phonological processing. Considered to be the most influential explanation, the phonological theory suggests that dyslexia is an outcome of a phonological awareness deficit. For instance, dyslexic children are unable to do tasks that require segmenting words into smaller units (syllables and phonemes).

Problems in memory are manifested in the areas of short-term verbal memory processing, working memory and visual memory; however, naming and articulation are typically within normal limits.

The notion of deficits in perception (visual-perceptual, visual-spatial or visual-motor) as underlying factors in reading disorder is represented, for instance, by letter reversals and spatial orientation confusion. When instructed to sequentially order letters, individuals can see a combination of letters but cannot perceive them in the correct order. Moreover, slow perceptual processing has been targeted as the core of dyslexia (e.g. [2]).

Lastly, dyslexia has been linked to attention difficulty. Researches point to rapid processing problems as a secondary contributor to the disorder, which is particularly associated with an impaired ability to switch attention quickly. As stated in the sluggish attention hypothesis, unlike their normal-reading counterparts, it is difficult to disengage attention of dyslexics once they become focused [3], [4]. However, attention shifting can occur at different levels of cognitive processing but it has yet to be established clearly at what level (central and/or peripheral) is attention shifting impaired among dyslexics [5]. Moreover, in a serial task administered by Romani, Tsouknida, di Betta and Olson, adult dyslexics, despite having reduced attention capacity, were able to shift attention [6]. Despite anecdotal evidence that children with dyslexia have difficulty "keeping on track," comparably fewer studies have focused directly on the evidence of attention disorder.

In addition, multimodal processing of perceptual stimuli is 
generally inefficient among the reading-disabled. Their non-linguistic auditory perceptual deficits (such as difficulties in discrimination between acoustically-similar sounds) are likely to be related to an inability to focus auditory attention in distinguishing properly and rapidly the features of the relevant signal sound [4]. Also proposed is that a causal link exists between reading deficits and phonologic problems involving the capture of auditory and visual automatic attention. There is evidence pointing to the presence of a cross-modal letter-to-speech sound integration deficit [7]. Facoetti, Lorusso, Cattaneo, Galli and Molteni suggest that focused spatial attention (FSA), a crucial factor affecting multimodal perceptual processing efficiency, enhances neural representation of the attended stimuli by faster reaction times, improved sensitivity and reduced flanker effect [8]. When one is flooded with much sensory information, it is possible to focus attention on one spatial location (or/and object) and to process the relevant information. It also allows decisions to be based on the stimulus alone by disregarding distracting stimuli. Individuals with reading problems suffer from a sluggish FSA, accounting for the generally inefficient multimodal processing of perceptual stimuli [8].

Dichotic listening, a non-invasive technique used to assess lateralization and cognitive functions, such as attention, involves the simultaneous presentation of two conflicting stimuli to both ears (e.g., [9]). Individuals monitor and shadow the message in one ear while ignoring the message presented in the other ear, thus measuring the brain's ability to process linguistic and non-linguistic information. Only one stimulus is to be reported. Commonly-used auditory materials are in the form of digits, words, consonant-vowel (CV) syllables and sentences. However, the most popular is the use of the CV stimulus set (stop consonants, such as /g/, $/ \mathrm{p} /$ and $/ \mathrm{t} / \mathrm{which}$ are paired with the vowel /a/) since it elicits the most robust right ear effects [10]. The present-day version of the dichotic listening technique was developed by Kimura in 1961 which consists of a three-stage mode of response: the Non-Forced Attention (NF), the Forced Right (FR) and the Forced Left (FL) [11]. In the NF, the subject is given no special instruction to direct attention but is simply asked to immediately repeat whichever sound is heard more clearly. This is the most common paradigm. To control for strategy effects and to study the role of dynamic cognitive factors, such as attention, the Forced Conditions (FR and FL) are administered. Also known as Directed or Focused Attention, these involve directing attention towards and reproducing the stimulus presented to the right (FR) or the left (FL) ear.

As a consequence, three kinds of ear advantage results can be derived, based on the greater proportion of stimuli correctly reported favoring one ear. The Right Ear Advantage (REA) indicates left hemisphere language dominance. Having an REA for verbal stimuli (CV syllables) reflects normal speech lateralization. On the other hand, a Left Ear Advantage (LEA) for verbal stimuli implies a less activated left hemisphere and a failure to suppress the right hemisphere during verbal tasks. Lastly, a No Ear Advantage (NEA) points to an unclear language dominance or an equal or bilateral performance between the two ears.

This investigation involving a small group of participants focuses on the evaluation of attention among normal reading children and children with dyslexia through their reported ear advantage results. It aims to answer whether children with dyslexia manifest problems in bimodal attention shifting. It is hypothesized that, due to difficulties in switching attention and in multi-modal processing, participants with dyslexia will report lesser REAs, especially in the Visual-Dichotic Listening (ViDiLi) Conditions. This will indicate a greater focus is on the visual cues despite hearing auditory stimuli. For this purpose, the ViDiLi program [12] was specifically developed, combining these two bimodal stimuli.

\section{METHOD}

\section{A. Participants}

Twelve pupils (10-11 years) from three private schools-two special education (SPED) and one non-graded mainstream - were selected. Six pupils with reading problems ( $M=10.6$ years; 4 males) enrolled in SPED and six normal-reading pupils $(\mathrm{M}=10.83$ years; 4 males) from a mainstream school were matched based on IQ, age and gender. Intelligence was measured using the Test of Nonverbal Intelligence -3 . Only those with at least average scores were included. Reading skills were assessed using the Reading comprehension subtest of the Wide Range Achievement Test - 4. Children with standard scores of 89 and below (below-average to lower extreme) comprised the group with reading problems $(M=78.67 ; \mathrm{SD}=7.45)$. For the control group (normal readers), participants had standard scores of 90 and above (average to upper extreme) in the reading subtest $(\mathrm{M}=99.33 ; \mathrm{SD}=6.02)$.

In addition, pupils from SPED had been previously evaluated by another professional (i.e., clinical psychologist, special educator) as having a reading disability. Children from the mainstream school had average academic performance based on teacher reports and academic records.

All of the participants were right-handed. Years of schooling were from five to seven years. Based on school records, parent- and teacher- interviews and observations, no child had a history of recurrent ear infections, severe hearing problems and uncorrected vision problems. Likewise, there was no diagnosis of attention-deficit hyperactivity disorder, intellectual disability, emotional and behavioural problems and developmental pervasive disorder.

\section{B. Data Gathering Procedure}

As a standard procedure when conducting studies involving elementary schools, the researcher asked permission from the Department of Education. After meeting with a number of school administrators, two SPED institutions and one mainstream school allowed their pupils to take part in the research. The individually-administered intelligence and reading achievement tests were done in a quiet room in the respective participant's school. On separate testing dates, the ViDiLi Program was conducted. During all of the sessions, visual and auditory distractions were controlled.

\section{Materials}

Reading ability: Wide Range Achievement Test-Fourth Edition [13]. WRAT-4 measures the basic academic skills of word reading, sentence comprehension, spelling and math 
computation. This test has alternate forms (Blue and Green), which can be used interchangeably with comparable results. Reliability evidence is high. In the age-based sample of the Alternate-Form Immediate Retest Reliability, coefficients ranged from .78-.89 while in the grade-based sample, it ranged from .86-.90. For the purpose of this research, only the Word Reading and Sentence Comprehension (reading) subtests of the Blue Form were administered. The combined standard scores of these two subtests make up the Reading Composite score, which was used in participant selection.

Nonverbal intelligence: Test of Nonverbal Intelligence, $3^{\text {rd }}$ edition [14]. TONI-3, a non-verbal test, can be used from ages 6 years, 0 months to 89 years, 11 months. It assesses intelligence, aptitude, abstract reasoning and problem solving and does not require reading, writing, speaking or listening on the part of the examinee, making it suitable for individuals who were diagnosed or believed to have communication disorders, such as learning disabilities. It is individually administered which takes up between 15-20 minutes of testing time. It has two equivalent forms (A and B), each consisting of 45 items. It provides a composite score of non-verbal intelligence. Interrater-reliability is high at .99 while alternate-form reliabilities ranged from .74 to .95 .

Attention: Dichotic Listening with Consonant-Vowel (DL CV) Syllables CD [15]. This was used to determine the baseline ear advantage (BEA) of both groups. Auditory stimuli, in the form of CV monosyllables, consists of the six stop-consonants combined with the vowel a. These are presented simultaneously to both ears. Participants are instructed to say which syllable (either from the left or right ear) they heard was the louder. The six syllables resulting from these combinations are paired, totaling to 36 pairs or trials. Stimuli were recorded using a male voice, maintaining constant intonation and intensity throughout the procedure. Each presentation of a pair of syllable lasts roughly $350 \mathrm{~ms}$, with an interval in-between presentation of approximately 4 seconds. Total run time is approximately 4 minutes. Test-retest reliability is between .70 to .80 while validity is between .70 to .80 [16]. This program was used to determine the baseline (initial) ear advantage of both groups. The CD was played through headphones connected to a laptop.

Visual-Dichotic Listening Bimodal Tasks CD [14]. The ViDiLi (using JAVA) was specifically developed for the study to assess the attentional ability of the participants. It is comprised of two tasks - the Non-Forced DL with Visual Cues and the Guided DL. In both of these tasks, a visual cue (arrow) was presented at the center of the screen prior to the presentation of the auditory stimuli.

In the Non-Forced DL with Visual Cues, instructions are similar to DL CV. No mention of the arrow's presence is given. On the other hand, for the Guided DL, participants are told to say the syllable from the particular ear side as directed by the arrow. This is more complicated than the previous dichotic listening conditions, since participants had to accomplish three related tasks: (a) focus on the arrow at the center of the monitor; (b) remember the direction of the arrow; and, (c) say the CV syllable presented to the particular ear.

Running time is approximately 7 minutes. Tasks presented used the same CV syllables but included visual cues at the middle of the computer screen. It consists of 72 trials. In addition to the set of headphones for the participant, two computer screens were also used - one each for the participant and the experimenter.

\section{RESULTS}

Significant difference in the baseline (initial) ear advantage results. As measured by the free recall DL, initial ear advantage varies among the children with reading problems while ear advantage (REA) remains stable among the normal readers. A reduced number of REAs among the participants with reading problems is observed. Using the Mann Whitney U-Test, significant differences exist in the baseline ear advantage between groups $\left(\mathrm{U}=5 ; \mathrm{CV}_{\alpha .05}=5\right)$.

Significant difference between groups in the ear advantage in the ViDiLi Non-forced Condition. Majority in the reading problem group report LEAs while REA is dominant among the normal readers. Scores, in the form of ear advantage results, from the Non-Forced DL with Visual Cues, were rank-ordered. With a $U=1, \mathrm{CV}_{\alpha .05}=5$, a significant difference is indicated between groups, with the sum of ranks equal to 22 (with reading problems) and 56 (normal readers).

Significant difference between groups in the ear advantage in the ViDiLi Guided Dichotic Listening Condition. Ear advantage scores from the Guided condition were rank-ordered. With a $\mathrm{U}=4, \mathrm{CV}_{\alpha .05}=5$, significant difference is indicated between groups, with the sum of ranks equal to 53 (with reading problems) and 25 (normal readers). The group of normal readers reported an equal number of REAs and LEAs while majority from the reading problem group show REAs.

Significant difference between the BEA and the ear advantage in the ViDiLi Non-forced of the reading problem group. Based on the Wilcoxon T- test, significant difference is found between the baseline ear advantage and the ear advantage results in the ViDiLi Non-Forced for the reading problem group $\left(\mathrm{T}=0, \mathrm{CV}_{\alpha .05}=0\right.$, with ranks for positive values equal to 21 and ranks for negative values equal to 0 ). However, no significant difference was computed for the normal reader group.

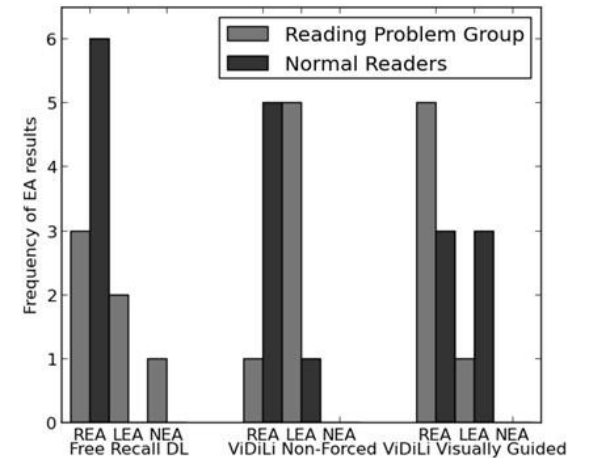

Fig. 1. Ear advantage results in the dichotic listening tasks.

Whether there is a significant difference between the BEA and the ear advantage in the ViDiLi Guided Dichotic Listening condition of the two groups, results showed no significant difference for both groups.

\section{DISCUSSION}

Since the left hemisphere is more dominant for language processing, REAs are generally reported for dichotic 
ally-presented verbal materials, such as CV syllables. Incidentally, such dichotic stimuli have shown to elicit the most robust ear advantage. Among normal-reading children, there is an existence of strong and consistent REAs. Results from this study likewise reflect stable REAs in the normal readers group, both in the baseline ear advantage and in the ViDiLi-Non forced DL. On the other hand, a decrease in their REAs in the ViDiLi Guided DL implies that ear advantage may be modulated by instructions and that attention to stimuli may shift. Despite the fact that REAs is a 'robust experimental phenomenon,' it can be overcome by direction to focus attention to one ear [17].

However, for children with reading problems, the ear advantage effect is unstable and changeable, implying a weak language processing by the left hemisphere during dichotic tasks. Due to phonological deficits, language-processing problems exist in the area of speech-sound discrimination (e.g., [18]). Lacking phonological awareness, poor readers are unable to efficiently segment words into phonemes, thereby failing to detect subtle differences between acoustically similar phonemes (e.g., /ba/ vs. /da/). In the investigation, children with reading problems likewise reported less REAs in the initial dichotic listening task and in the ViDiLi-Non-forced DL.

The greater variability in the ear advantages reported by the reading problem group in the ViDiLi Bimodal Tasks may indicate the influence of visual cues in their ability to switch attention. In the Non-forced DL with Visual Cues, wherein no mention of the purpose of the arrows was made in the instructions, children with reading problems reported more LEAs as compared to their baseline ear advantage. Such "implicit manipulation" elicited by the presence of the arrows may have influenced the group to focus more on the visual cues during this listening task. This may also indicate an inability to pay attention to the auditory stimuli and to be easily affected by the "non-relevant" stimulus (arrow). This may also point out to a failure to integrate bimodality-in this case, auditory and visual cues. In learning to read, performance in cross-modal abilities is a predictor [11]. Multimodal processing of perceptual stimuli is generally inefficient among individuals with reading problems as evidenced by a slower multimodal (auditory and visual) attention capture [19]. A sluggish attention shifting makes it more difficult for them to disengage from their focus, as compared to normal readers. In actual reading, children with reading problems exhibit deficiencies in the cross-modal shifting of attention (seeing the letter/word (vision), hearing the letter/word being pronounced (audition), pronouncing the letter/word (speech)).

On the contrary, when the presence of the arrow was explicitly mentioned in the instructions (ViDiLi Guided DL), they reported more REAs. Stating the purpose of the visual cues in relation to the $\mathrm{CV}$ variables may have enabled them to listen more to the auditory stimuli; thereby, activating the left hemisphere to a greater extent than in the previous conditions, leading to an increase in REAs. Furthermore, the explicitly-stated instructions may have served as their 'external' guide to prepare themselves to shift or switch attention from focusing on the visual stimuli to paying more attention to the auditory set, thereby activating to a greater extent the left hemisphere.
In previous studies involving cued versus non-cued tasks, learning disabled children, such as those with reading difficulties, are able to shift their attention with the instructional set in during dichotic listening. In addition, visual pre-cuing has been found to aid subjects in processing and identifying items mainly within the right, as opposed to the left, ear [20]. Although attention capacity may be deficient, individuals with dyslexia are able to shift attention [6].

\section{CONCLUSIONS}

The motivation for this research is to look into the attention abilities of pupils with and without reading problems as determined by the ear advantage. Attention was assessed using the ViDiLi program, which combines visual and auditory stimuli.

With regard to the findings, the author wishes the readers to keep in mind the following limitations of the study: Due to the qualifications specified by the researcher as suggested by literature on reading disabilities, only a limited number of children with "pure" reading problems (i.e., absence of co-morbid conditions, such as ADHD) qualified. Despite the increasing awareness of reading and other learning disabilities in Manila, assessment (and identification) of the condition is still not available widespread. The study also primarily focused on the differences in ear advantage and attention between children with and without reading problems. Correlations regarding the ear advantage and the ViDiLi conditions were not determined. In addition, reaction times when reporting the ear advantage were not recorded. As a result, the study did not compare the length of time it took for the participants to respond. Also, participants' memory abilities were not measured.

\section{ACKNOWLEDGMENT}

The author wishes to thank Mr Jó Ágila Bitsch Link (RWTH Aachen University, Germany) for co-developing the ViDiLi Program and Mr Mark Gale, Mr Reuben Nuval, and Ms Annabelle Jay-Ar Trono (University of Santo Tomas, Manila, Philippines) for their valuable assistance during data gathering. The schools in the city of Manila and Quezon City as well as the participants and their families, are gratefully acknowledged.

\section{REFERENCES}

[1] J. Driver, M. Eimer, and E. Macaluso, "Cross-modal consequences of human spatial attention," in Neurobiology of attention, L. Itti, G. Rees, and J. Tsotsos, Ed. Amsterdam: Elsevier Academic Press, 2005, pp. 187-196.

[2] P. Stenneken, J. Egetemeir, G. Schulte-Körne, H. J. Müller, W. X Schneider, and K. Finke, "Slow perceptual processing at the core of developmental dyslexia: a parameter based assessment of visual attention," Neuropsychological, vol. 49, no. 12 , pp. 3454-3465, Oct. 2011.

[3] J. A. G Lum, G. C. Ramsden, and A. K. Lindell, "The attentional blink reveals sluggish attentional shifting in adolescents with specific language impairment," Brain and Cognition, vol. 63, pp. 287-295, Apr. 2007.

[4] R. Hari and H. Renvall, "Impaired processing of rapid stimulus sequences in dyslexia," Trends in Cognitive Science, vol. 5, pp. 525-532, Dec. 2001.

[5] G. Stoet, H. Markey, and B. Lopez. "Dyslexia and attentional shifting," Neuroscience Letters, vol. 427, no. 1, pp. 61-65, Oct. 2007. 
[6] C. Romani, E. Tsouknida, A. M. di Betta, and A. Olson, "Reduced attentional capacity, but normal processing speed and shifting of attention in developmental dyslexia: Evidence from a serial task," Cortex, vol. 47, no. 6, pp. 715-733, June 2011.

[7] S. Franceschini, S. Gori, M. Ruffino, K. Pedrolli, and A. Facoetti, "A causal link between visual spatial attention and reading acquisition," Current Biology, vol. 22, pp. 814-819, May 2012.

[8] A. Facoetti, M. L. Lorusso, M. Cattaneo, K. Galli, and M. Molteni, "Visual and auditory attentional capture are both sluggish in children with developmental dyslexia," Acta Neurobiologiae Expermentalis, vol. 65 , pp. $61-72$, Oct. 2005

[9] M. Andersson, I. Reinvang, E. Wehling, K. Hugdahl, and A. J. Lundervold, "A dichotic listening study of attention control in older adults," Scandinavian Journal of Psychology, vol. 49, no. 4, pp. 299-304, Aug. 2008.

[10] K. Hudgdahl, "Brain lateralization: dichotic studies," In B. Smith and G. Adelman (Eds.), Encyclopedia of Neurosciences: Neuroscience Year 2. Boston, MA: Birkhauser, 1992.

[11] K. Hugdahl, "What can be learned about brain function in dichotic listening? Qué se puede Aprender sobre el Funcionamiento del Cerebro a partir de la Escucha Dicótica?" Revista Espanola de Neuropsicología vol. 2, no. 3, pp. 62-84, Sept. 2000.

[12] R. M. Ramos and J. A. Bitsch Link, Visual-Dichotic Listening (ViDiLi) Program, Computer Software, Germany, 2008.

[13] G. S. Wilkinson and G. J. Robertson, Wide Range Achievement Test 4 Professional Manual, Lutz, FL: Psychological Assessment Resources, 2006.

[14] L. Brown, R. J. Sherbenou, and S. K. Johansen, Test of Nonverbal Intelligence 3 professional manual, Austin, TX: PRO-ED, 1997.

[15] K. Hugdahl and K. Bronnick, Dichotic Listening CD, Norway: University of Bergen, 1988

[16] K. Hugdahl and A. Abjornsen, Dichotic Listening with CV-Syllables Manual, Norway: University of Bergen, 1988.

[17] K. Hugdahl, I. Law, S. Kyllingsbaek, K. Bronnick, A. Gade, and O. Paulson, "Effects of attention on dichotic listening: An ${ }^{15} \mathrm{O}-\mathrm{PET}$ study," Human Brain Mapping, vol. 10, pp. 87-97, June 2000.
[18] F. R. Manis, C. M. Chang, M. S. Seidenberg, P. Keating, L. M. Doi, B. Munson, and A. Petersen, "Are speech perception deficits associated with developmental dyslexia?" Journal of Experimental Child Psychology, vol. 66, no. 2, pp. 211-235, Apr. 1997.

[19] S. A. Rose, J. F. Feldman, J. J. Jankowski, and L. R. Futterweit, "Visual \& auditory temporal processing, cross-modal transfer, and reading," Journal of Learning Disabilities, vol. 32, pp. 256-266, May 1999.

[20] M. Hiscock, and C. K. Hiscock, "An anomalous sex difference in auditory laterality," Cortex, vol. 24, no. 4, pp. 595-599, Dec. 1988.

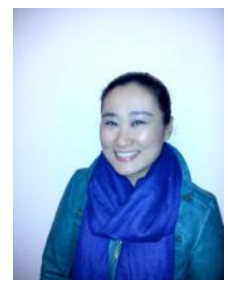

Roann M. Ramos graduated with a degree in BS Psychology from the University of Santo Tomas (UST), Manila, Philippines, in 1995 and received her M.A. in clinical psychology from the same University in 1998. Supported by a scholarship by the KAAD (German Catholic Academic Exchange Service), she participated in a sandwich program to pursue her $\mathrm{PhD}$ in Tübingen, Germany and the Philippines. She obtained her $\mathrm{PhD}$ in clinical psychology in 2008

Having started out her career as a guidance counselor, she is currently an Associate Professor of psychology at the College of Science and the Graduate School of UST. She was affiliated with the Neurodevelopmental Team of the UST Hospital (as clinical psychologist) and the Research Cluster on Culture, Education and Social Issues (as researcher). Her research activities are focused on, but not limited to, dyslexia, shadow education, and cyber psychology. She recently published a mobile app, Psychologist in a Pocket, together with RWTH Aachen University, Germany, helping psychologists and other mental health professionals to quickly and conveniently screen depression through the analysis of patients' electronic data.

Dr. Ramos is a member and a Certified Clinical Psychologist of the Psychological Association of the Philippines (PAP). Her other memberships include the International Dyslexia Association, the Autism Society and the American Psychological Association (APA) Division 35 